\title{
Estudo prospectivo de pacientes pediátricos com dor abdominal crônica
}

\author{
Prospective study of infants with chronic abdominal pain
}

Tatiana Kores Dorsa1 , Gabriel Hessel2 ${ }^{2}$ Magno Cardoso V. Neto ${ }^{3}$, Elizete Aparecida L. C. Pinto ${ }^{2}$

\section{RESUMO}

Objetivo: Classificar a dor abdominal crônica em crianças e adolescentes por meio dos critérios de Roma II e definir o desfecho diagnóstico em três anos de seguimento.

Métodos: Durante um ano, 71 pacientes com dor abdominal crônica foram atendidos como casos novos num ambulatório terciário de gastroenterologia pediátrica. Causas orgânicas foram excluídas por bases clínicas e laboratoriais, e relatos clínicos foram avaliados especificamente quanto à possibilidade de preencherem os Critérios de Roma II para dor abdominal em crianças. Para estabelecer o diagnóstico definitivo, os pacientes foram seguidos por três anos, em média.

Resultados: A alocação dos 71 pacientes segundo Roma II foi: doença orgânica $(n=12)$, remissão dos sintomas após a primeira consulta $(n=7)$, ou preencheram os critérios para dor funcional $(n=52)$. Dos 12 pacientes de doença orgânica, nove foram diagnosticados como intolerantes à lactose, mas foram re-alocados para doença funcional no seguimento, visto que a dieta de isenção não aliviou a queixa. Dos 52 pacientes com doença funcional (idade mediana $=9,3$ anos, $50 \%$ meninos), nove, que inicialmente preencheram o critério para dor abdominal funcional, foram re-alocados no diagnóstico de constipação funcional e 43 mantiveram o diagnóstico funcional: 24 com dispepsia funcional, 18 com dor abdominal funcional e um com síndrome do intestino irritável.

Conclusões: Dentre os casos de dor abdominal crônica, a dor do tipo funcional foi mais comum que as causas orgânicas e, dentre os seus subgrupos, a dispepsia funcional foi mais freqüente. $\mathrm{O}$ seguimento em longo prazo permitiu estabelecer o diagnóstico definitivo da origem da dor abdominal nessas crianças.

Palavras-chave: dor abdominal; síndrome do intestino irritável; criança.

${ }^{1}$ Residente de Pediatria do Hospital das Clínicas da Universidade Estadual de Campinas (Unicamp)

${ }^{2}$ Doutor em Saúde da Criança e do Adolescente, professor assistente de Gastroenterologia Pediátrica da Faculdade de Ciências Médicas (FCM) da Unicamp

${ }^{3}$ Mestre em Saúde da Criança e do Adolescente pela FCM-Unicamp

Endereço para correspondência:

Elizete Aparecida L. C. Pinto

Estrada da Rhodia, 7.250 - Condomínio Residencial Lagoa Serena,

\section{ABSTRACT}

Objective: To classify chronic abdominal pain in children and adolescents, according to Rome II criteria and to define diagnosis outcome in a three-year follow-up period.

Methods: During one year, 71 consecutive new patients with abdominal pain were seen in a tertiary pediatric gastroenterology outclinic. Organic diseases were excluded by physical examination and laboratory procedures, and clinical complaints were evaluated according to Rome II criteria for children abdominal pain. In order to establish definitive diagnosis, patients were followed for a mean of three years.

Results: Allocation of the 71 patients was: organic disease $(\mathrm{n}=12)$, complete remission of symptoms following first clinical presentation ( $n=7)$, and functional disease $(n=52)$. Nine patients from the organic disease group, who had been diagnosed as having lactose intolerance, were re-allocated to the functional disease criteria since their symptoms did not relieve when lactose was excluded from their diet. Out of the 52 patients of the functional disease group (median age $=9.3$ years, $50 \%$ boys), nine children with initial diagnosis of functional abdominal pain were re-allocated to functional constipation diagnosis during follow-up, and other 43 patients fulfilled the functional disease diagnosis defined by Rome II: 24 with functional dyspepsia, 18 with functional abdominal pain and one with irritable bowel syndrome.

Conclusions: In a group of children with chronic abdominal pain, functional diseases were found more frequently than organic diseases, and functional dyspepsia was the commonest subtype. Long term follow-up was useful to establish definitive diagnosis in children with chronic abdominal pain.

Key-words: abdominal pain; irritable bowel syndrome; child.

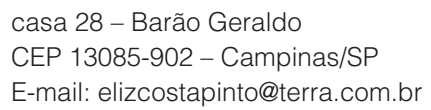

Fonte financiadora: Programa Institucional de Bolsas de Iniciação Científica do Conselho Nacional de Desenvolvimento Científico e Tecnológico (Pibic/CNPq)

Recebido em: 6/3/2007

Aprovado em: 22/5/2007 


\section{Introdução}

A dor abdominal recorrente constitui uma queixa freqüente na população pediátrica e é responsável por cerca de 2 a $4 \%$ das consultas ${ }^{(1)}$. A prevalência relatada em escolares varia de 7 a $25 \%$ em estudos de campo ${ }^{(2-4)}$. Apley e Naish, em 1958, descreveram a dor abdominal recorrente como uma entidade nosológica que incluía dor abdominal de etiologia funcional ou orgânica ${ }^{(2)}$, ocorrendo em um grupo heterogêneo de doentes com diferentes características clínicas. No entanto, desde então, não foi delineado um fluxograma de investigação ou tratamento para a condição. Com o avanço das técnicas diagnósticas, o termo "dor abdominal recorrente" passou a representar dor abdominal de origem não orgânica, diagnosticada pela exclusão de causas identificáveis por testes laboratoriais. Em 1999, os critérios de Roma II para doenças funcionais foram elaborados para definir o diagnóstico da dor abdominal crônica em crianças ${ }^{(5)}$. Quando os sintomas de pacientes com dor abdominal correspondem rigorosamente aos critérios de Roma preconizados para os diagnósticos de dispepsia funcional, síndrome do intestino irritável, dor abdominal funcional e migrânea abdominal, o diagnóstico definitivo destas entidades pode ser estabelecido, sem necessidade de investigação laboratorial adicional.

A utilidade do critério de Roma II como um instrumento para o diagnóstico de dor abdominal na prática clínica não foi testada em crianças brasileiras. Este estudo teve por objetivo registrar o diagnóstico etiológico da dor abdominal crônica em crianças atendidas num serviço terciário, identificando a presença de causas orgânicas ou a alocação nos subgrupos do critério Roma II, além de verificar a pertinência do diagnóstico no seguimento do paciente por um período médio de três anos.

\section{Métodos}

Estudo longitudinal, prospectivo e descritivo. Foram elegíveis todas as crianças com dor abdominal crônica, atendidas como casos novos no ambulatório de Gastroenterologia Pediátrica de um Hospital terciário, no período de agosto de 2001 a julho de 2002. Foram incluídas no estudo as crianças cuja queixa principal preencheu a definição de Hyams et al $l^{(6)}$ para dor abdominal, ou seja, pelo menos três episódios de dor abdominal que tivessem afetado as atividades da criança no período de pelo menos três meses. O trabalho foi aprovado pela Comissão de Ética do Hospital das Clínicas da Universidade Estadual de Campinas (Unicamp).
Foram excluídos do estudo pacientes com idade inferior a quatro anos e aqueles sabidamente portadores de quaisquer diagnósticos adicionais de origem física ou psicológica ou, ainda, aqueles com antecedente cirúrgico.

Após diagnóstico inicial de dor abdominal recorrente ${ }^{(6)}$, a investigação adicional e o tratamento eram indicados a critério de um gastroenterologista pediátrico que acompanhou o diagnóstico e o seguimento de todos os pacientes até a conclusão do período de estudo.

$\mathrm{Na}$ consulta inicial, um gastroenterologista pediátrico aplicou um questionário estruturado a todos os pacientes. Este questionário constitui uma versão modificada do Questionário para Doença Intestinal ${ }^{(7)}$, um instrumento validado e usado extensivamente para as doenças gastrointestinais funcionais em adultos, adicionando-se elementos do Questionário para os Sintomas Gastrintestinais na Pediatria ${ }^{(8)}$, que visa identificar sintomas associados a doenças gastrointestinais em crianças de acordo com os critérios de Roma $\mathrm{II}^{(5)}$. Os questionários foram aplicados após assinatura de termo de consentimento livre e esclarecido.

Foram questionadas informações referentes à duração, localização, freqüência, natureza, fatores desencadeantes e de alívio da dor, bem como possíveis relações com a alimentação e evacuação. O questionário também incluiu uma descrição detalhada do hábito intestinal do paciente. Informações adicionais relacionadas a queixas gastrointestinais (eructação, náuseas, vômitos, regurgitação, distensão abdominal) e extra-intestinais (cefaléia, aura e sintomas urinários) também foram solicitadas. O questionário abrangeu detalhes sobre o crescimento e desenvolvimento, avaliações e tratamentos prévios, história compatível com cólicas do lactente, diarréia funcional e refluxo gastroesofágico. A freqüência de faltas escolares associadas à dor abdominal também foi anotada.

No exame físico, atenção particular foi dispensada ao exame do abdome, à presença de fezes impactadas ao toque retal (presença de fezes na ampola retal) e à inspeção da área perineal.

Avaliação laboratorial padronizada foi realizada em todos os pacientes e incluiu hemograma, velocidade de hemossedimentação, exame do sedimento de urina e urocultura, exame parasitológico de fezes, dosagem de aminotransferases e eletroforese de proteínas. Foram ainda realizados os seguintes exames para investigação de doença orgânica: endoscopia digestiva alta com biópsias esofágica e gástrica, ultra-sonografia abdominal e pélvica, além do teste de tolerância à lactose. 
O estado nutricional dos pacientes foi avaliado no início do acompanhamento. Foram calculados os escores $\mathrm{Z}$ das seguintes relações antropométricas: peso/idade, estatura/idade e peso/estatura, por meio de comparação com os valores de referência do National Center for Health Statistics (NCHS) ${ }^{(9)}$.

Uma vez excluída doença orgânica, com base nos elementos diagnósticos descritos, os pacientes foram incluídos, se possível, em um dos cinco grupos distintos da dor abdominal, definidos pelos critérios de Roma II $^{(5)}$. Os pacientes foram acompanhados por um período médio de três anos. As crianças foram reavaliadas a cada três meses quanto à sua inclusão nos critérios de Roma II e quanto à possibilidade da existência de doença orgânica não identificada previamente.

As doenças funcionais são condições clínicas não associadas a anormalidades físicas ou fisiológicas, infecções, inflamações ou neoplasias. Podem ser diagnosticadas apenas com base nas queixas dos pacientes e não são disponíveis marcadores biológicos. Os sintomas são interpretados como tendo, em comum, distúrbios sensoriais ou motores do trato gastrointestinal. Os critérios de Roma foram desenvolvidos para o diagnóstico de doenças funcionais do trato gastrointestinal. A classificação de Roma II é baseada na premissa de que uma desordem funcional pode ser diagnosticada com base na identificação de informações referidas pelos pacientes. Em 1999, foram publicados os requisitos para o diagnóstico das doenças gastrointestinais pediátricas ${ }^{(5)}$ para serem usados como um instrumento diagnóstico e método de pesquisa. Definiram-se os requisitos para quatro tipos de sintomas: vômitos, dor abdominal, diarréia funcional e distúrbios da evacuação. Para cada tipo de sintoma, entidades clínicas foram definidas com base na presença de determinados sintomas. No grupo da dor abdominal, cinco entidades foram identificadas: dispepsia funcional, classificada em dispepsia do tipo úlcera, dismotilidade ou inespecífica; síndrome do intestino irritável; dor abdominal funcional; migrânea abdominal e aerofagia, de acordo com características particulares a cada subgrupo. O critério de Roma II foi reavaliado e atualizado recentemente com base em evidências científicas e experiência clínica. Em 2006, essa atualização foi publicada ${ }^{(10)}$ e os grupos sintomáticos de interesse foram re-classificados em: 1) síndrome dos vômitos cíclicos, ruminação e aerofagia; 2) sintomas relacionados com dor abdominal, incluindo quatro subgrupos: dispepsia funcional, síndrome do intestino irritável, migrânea abdominal e dor abdominal funcional e 3) constipação funcional e incontinência fecal não retentiva.

\section{Resultados}

No período de 12 meses em que se incluíram crianças no estudo, foram aplicados 71 questionários para pacientes com dor abdominal crônica (Figura 1). A mediana de idade dos 71 pacientes na primeira consulta foi 9,3 anos

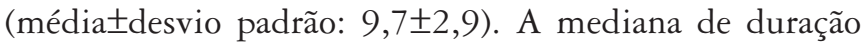
pregressa da queixa foi 3,5 anos (média \pm desvio padrão: $4,2 \pm 2,7)$. Foram estudados 37 pacientes do gênero masculino e 34 do feminino. De acordo com as medidas avaliadas na primeira consulta, os 71 pacientes apresentavam peso e altura adequados para a idade.

Causas orgânicas foram identificadas em 12 pacientes, a saber, refluxo gastroesofágico em um, gastrite péptica em um, má rotação intestinal em um e intolerância à lactose em nove.

Sete pacientes relataram remissão completa dos sintomas após a consulta inicial, sendo acompanhados clinicamente no período de avaliação da coorte. Os demais 52 pacientes preencheram os critérios de Roma II para doença funcional. A classificação de acordo com os subgrupos está apresentada na Figura 2.

Durante o acompanhamento dos 52 pacientes, identificou-se que, em nove, a queixa de dor abdominal estava associada ao diagnóstico de constipação intestinal funcional. Em virtude de mudança das respostas ao questionário, os critérios de diagnóstico da Sociedade NorteAmericana de Gastroenterologia e Nutrição Pediátrica ${ }^{(11)}$ e a definição proposta pelos critérios de Roma $\mathrm{II}^{(5)}$ para o diagnóstico de constipação passaram a ser preenchidos. A associação entre o quadro de dor abdominal e a constipação foi confirmada quando ocorreu o desaparecimento da dor abdominal em resposta ao tratamento da constipação (Figura 2).

Das 12 crianças inicialmente definidas como portadoras de doença orgânica, em nove delas tinha sido estabelecido o diagnóstico de intolerância à lactose com base em teste laboratorial. Entretanto, a clínica atribuída à má absorção de carboidratos manteve-se a despeito da aderência à dieta de isenção do açúcar. Estes pacientes prospectivamente preencheram o diagnóstico definitivo de síndrome do intestino irritável (Figura 2).

Dentre os pacientes que satisfizeram os critérios de Roma II para o diagnóstico de dor abdominal, nenhum apresentou doença orgânica no seguimento e o tratamento foi baseado em orientação familiar sobre o distúrbio. 


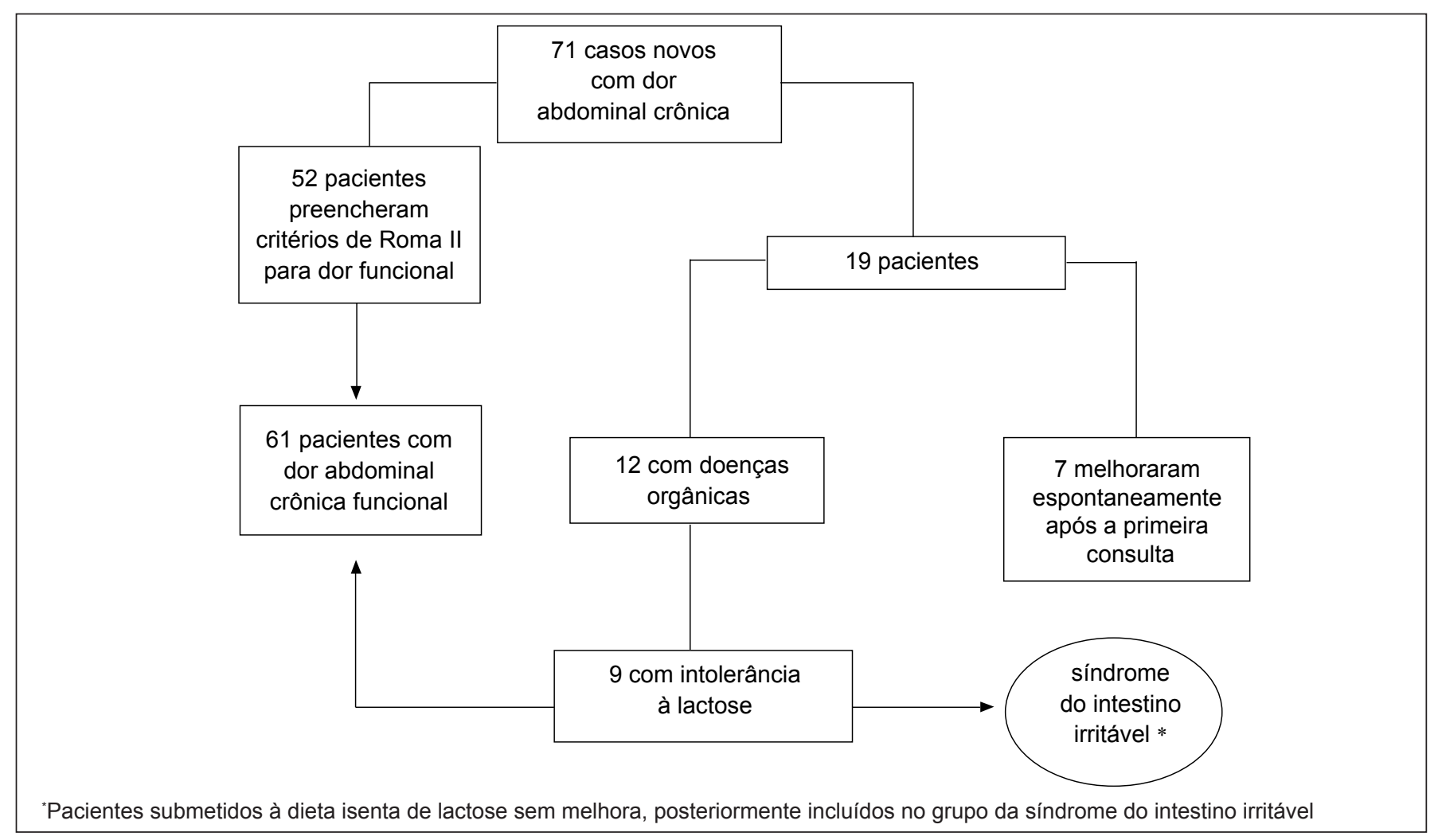

Figura 1 - Diagnóstico de 71 pacientes com dor abdominal crônica, admitidos como casos novos, no período de um ano, divididos em dor de origem orgânica ou funcional, classificada de acordo com os critérios de Roma II

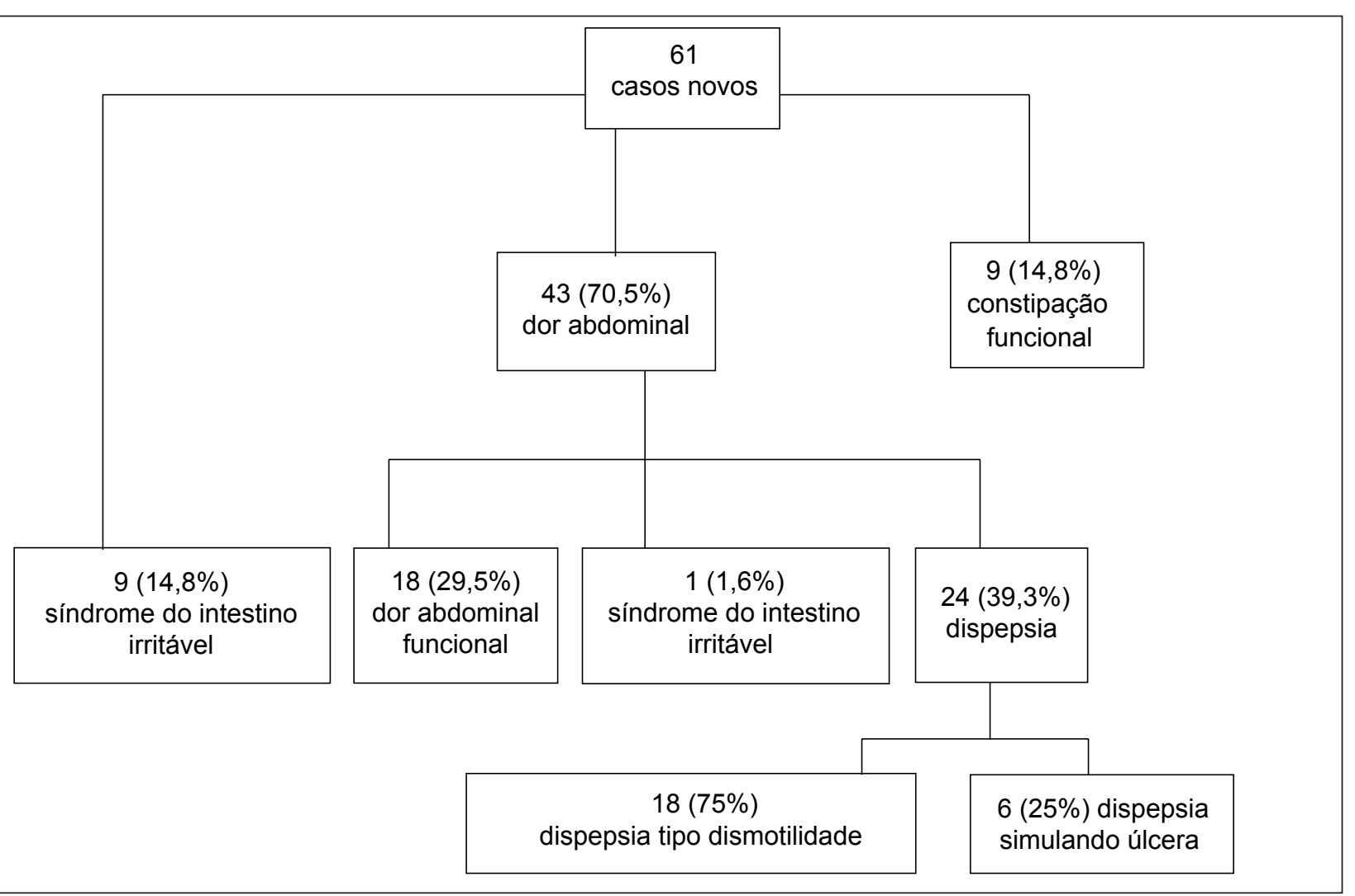

Figura 2 - Desfecho do diagnóstico de 61 casos novos com dor abdominal crônica, diagnosticados como tendo doença funcional, acompanhados por um período de três anos e classificados de acordo com os critérios de Roma II 


\section{Discussão}

Este é o primeiro estudo a investigar os resultados da aplicação do critério de Roma II para o diagnóstico da dor abdominal em crianças brasileiras. No período de 36 meses de seguimento para definição do diagnóstico, dos 71 casos novos de crianças com dor abdominal recorrente, a aplicação do critério de Roma identificou inadequadamente nove pacientes $(12,7 \%)$ como portadores de dor abdominal funcional, sendo que, no seguimento, tais diagnósticos foram redefinidos para constipação funcional. Em outros nove pacientes, o diagnóstico inicial foi intolerância à lactose, porém, não houve melhora clínica com a dieta de isenção de carboidrato, sendo, posteriormente, possível firmar o diagnóstico de doenças funcionais pelo preenchimento do critério de Roma. Nenhum paciente que recebeu o diagnóstico de doença funcional desenvolveu doença orgânica no seguimento.

Ao avaliar a abrangência dos resultados do presente estudo, três fatores devem ser considerados. O primeiro refere-se às características dos pacientes analisados, ou seja, crianças provenientes de Unidades Básicas de Atenção à Saúde, onde não se emprega o critério de Roma II para diagnosticar doenças funcionais e que foram referidas para um serviço terciário. Esses pacientes foram encaminhados ao serviço especializado porque suas queixas não puderam ser esclarecidas nos níveis básicos de atendimento à saúde e representam uma fração das crianças que, de fato, apresentam sintomas mais intensos e prolongados.

A segunda limitação tem base no critério de elegibilidade para o estudo. Foram selecionados pacientes cuja queixa principal era dor abdominal, excluindo-se aqueles pacientes com distúrbios funcionais semelhantes que apresentavam outras queixas predominantes, por exemplo, náuseas e/ou empachamento, que poderiam, sob outro critério de seleção, ser incluídos em outros subgrupos da dor abdominal funcional.

Finalmente, devem-se considerar as limitações inerentes ao uso de questionário para obtenção das informações iniciais e, ainda, possíveis diferenças entre a percepção dos pais, quando respondem a um interrogatório sobre a sintomatologia da criança, e o sintoma real ${ }^{(12)}$.

O critério metodológico para estabelecer o diagnóstico definitivo foi o tempo de seguimento. Em favor desse critério, dois aspectos devem ser considerados. Primeiro, o fato de que os pacientes realizaram ampla investigação laboratorial, incluindo a endoscopia digestiva, para afas- tar entidades nosológicas que poderiam cursar sem sinais clínicos de alarme. Em segundo lugar, verificou-se que, no período de seguimento de 36 meses, nenhum dos pacientes que preencheu um dos critérios para dor funcional evoluiu com prejuízo nos índices de crescimento.

$\mathrm{Na}$ faixa pediátrica, a desnutrição e a parada de crescimento são inevitáveis em condições clínicas que cursem com aumento das perdas de nutrientes por via digestiva ou outra via, aumento do consumo de energia, diminuição da síntese de proteínas secundária à insuficiência hepática, interferência com a incorporação de nutrientes e obstrução anatômica ou funcional do tubo digestivo. Considerando que não foram identificados sinais de alarme ou prejuízo no crescimento durante o seguimento e até porque, se assim fosse, o diagnóstico de doença funcional seria imediatamente descartado; considerando também que os trabalhos que se dedicaram à validação dos critérios de Roma em pediatria limitaram-se a um tempo de seguimento máximo de dois $\operatorname{anos}^{(13-15)}$; e, finalmente, que o objetivo não foi estabelecer a história natural da doença funcional, o período de seguimento de três anos foi considerado adequado.

A constipação intestinal deve ser avaliada com atenção no diagnóstico diferencial da dor abdominal recorrente em escolares. A dificuldade para obter informações relacionadas às características das fezes e do ato evacuatório pode estar relacionada ao fato de que o escolar é independente nos seus hábitos intestinais e, em geral, é a mãe quem responde aos questionamentos médicos. Quando as queixas são referidas, é importante considerar não só a freqüência das evacuações, mas também as características das fezes e a presença de dificuldade e/ou esforço durante a evacuação. Deve-se lembrar, ainda, que a associação de ambos os diagnósticos é também uma outra possibilidade biologicamente plausível ${ }^{(16)}$.

A condição de constipação sem escape fecal na faixa etária de escolares e adolescentes não foi contemplada nos diagnósticos definidos pelos critérios pediátricos de Roma II para constipação funcional, condição que foi reavaliada na nova versão dos critérios, Roma III ${ }^{(10)}$. O comitê que elaborou o Roma II dividiu os tipos de constipação em: 1) disquesia do lactente; 2) constipação funcional e 3) retenção fecal funcional. Essa subdivisão não considerou a possibilidade de que pacientes poderiam ser incluídos indistintamente nos subgrupos 2 e 3, e, ainda, que pacientes poderiam mudar do subgrupo 2 para o 3 na evolução da doença. O critério de Roma III fundiu as duas condições em uma só categoria denominada de constipação funcional. 
No presente estudo, a dispepsia do tipo dismotilidade foi o subgrupo clínico de dor abdominal mais comumente encontrado. O sintoma dispepsia pode estar presente em uma série de outras doenças, tais como: esofagite, doença péptica, doença inflamatória intestinal, doença do trato biliar e hepática, intolerância à lactose e constipação intestinal ${ }^{(17)}$. A baixa especificidade torna a avaliação da dispepsia mais difícil, necessitando, na maioria das vezes, de realização de endoscopia digestiva alta. Hyams et al ${ }^{(18)}$ acompanharam por dois anos 127 crianças que preenchiam os critérios de Roma II para dispepsia e verificaram que pacientes com alterações leves a moderadas na endoscopia tiveram bom prognóstico, independentemente de receberem ou não tratamento. $\mathrm{Na}$ ausência de sinais de alarme, o resultado da avaliação endoscópica não influenciou o prognóstico dessas crianças. Uma hipótese provável é a de que as anormalidades mucosas encontradas em alguns exames não estivessem relacionadas aos sintomas.

É possível que muitas crianças sejam excluídas indevidamente do diagnóstico de doença funcional, caso se considere rigorosamente o critério de Roma II, no qual qualquer anormalidade mucosa constituiria critério de exclusão. Na versão Roma III, o comitê eliminou a obrigatoriedade de realizar a endoscopia digestiva alta para firmar o diagnóstico de doença funcional, considerando que, em crianças, a possibilidade de anormalidades da mucosa associadas a sintomas dispépticos não é significativa.

Nos estudos internacionais recentes que procuraram distinguir os diferentes tipos de doença funcional com predomínio de dor abdominal em crianças $^{(4,18,19)}$, foram identificados com maior freqüência os grupos de crianças com sintomas relacionados à síndrome do intestino irritável (30 a 50\% dos casos) e dispepsia (15 a 50\% dos pacientes). A presente casuística diferiu das séries internacionais quanto à freqüência de pacientes com síndrome do intestino irritável, que varia de 68 a $40 \%$ dos $\operatorname{casos}^{(4,20,21)}$. Neste estudo, a primeira avaliação permitiu incluir apenas um paciente neste diagnóstico. Esta diferença na prevalência da síndrome do intestino irritável poderia ser explicada por inabilidade das crianças em relatar as características dos sintomas ou por interpretação errônea dos pais ou cuidadores, cujas informações podem ter sido influenciadas pelas suas próprias histórias médicas ou, ainda, por erro na aplicação do questionário. Contudo, no seguimento, mais nove pacientes puderam ser incluídos nesse critério.

A hipolactasia do adulto é uma condição freqüente na população brasileira; 28 a $100 \%$ das crianças investigadas nos estudos epidemiológicos em nosso meio apresentam testes de tolerância à lactose alterados ${ }^{(22)}$. O teste de tolerância à lactose pode, reconhecidamente, superestimar o diagnóstico da intolerância ${ }^{(23)}$. A baixa prevalência de síndrome do intestino irritável poderia ser explicada pelo fato de que crianças com dor abdominal do tipo síndrome do intestino irritável terem sido excluídas da casuística de dor abdominal recorrente porque foram impropriamente diagnosticadas como portadoras de intolerância à lactose, com base nos seus exames laboratoriais que superestimaram o diagnóstico de intolerância e, ainda, pela possibilidade da ocorrência simultânea das duas condições. A combinação dos dois diagnósticos num mesmo paciente pode ter interferido na seleção clínica. Na série estudada por Gremse et al ${ }^{(19)}, 61 \%$ das 146 crianças avaliadas para dor abdominal foram incluídas no grupo da síndrome do intestino irritável. Todos estes pacientes realizaram o teste do $\mathrm{H}_{2}$ expirado para lactose e um terço apresentou teste positivo. $\mathrm{O}$ manejo dos pacientes com teste positivo por meio de dieta isenta de lactose não reduziu a freqüência da dor abdominal, no entanto, diminuiu significativamente a necessidade de medicação. Estes dados reforçam a possibilidade da concomitância dos diagnósticos ${ }^{(24)}$.

Uma das finalidades dos critérios de Roma é racionalizar o uso dos exames complementares na investigação das doenças funcionais. Contudo, na maioria dos critérios, evidencia-se a exclusão obrigatória de doenças metabólicas e estruturais. Não está claro, na elaboração dos critérios, até que ponto esta busca ativa por doenças orgânicas deve prosseguir nem, tampouco, se enfatiza que a melhor pista diagnóstica para doença funcional é o acompanhamento do paciente. 


\section{Referências bibliográficas}

1. Starfield B, Hoekelman R, McCormick M, Benson P, Mendenhall RC, Moyniham $\mathrm{C}$ et al. Who provides health care to children and adolescents in the United States? Pediatrics 1984;74:991-7.

2. Apley J, Naish N. Recurrent abdominal pain: a field survey of 1000 school children. Arch Dis Child 1958;33:165-70.

3. Oster J. Recurrent abdominal pain, headache, and limb pains in children and adolescents. Pediatrics 1972;50:429-36.

4. Hyams JS, Burke G, Davis PM, Rzepski B, Andrulonis PA. Abdominal pain and irritable bowel syndrome in adolescents: a community-based study. J Pediatr 1996;29:220-6.

5. Rasquin-Weber A, Hyman PE, Cucchiara S, Fleisher DR, Hyams JS, Milla PJ et al. Childhood functional gastrointestinal disorders. Gut 1999;45:S60-8.

6. Hyams JS, Treem WR, Justinich CJ, Davis P, Shoup M, Burke G. Characterization of symptoms in children with recurrent abdominal pain: resemblance to irritable bowel syndrome. J Pediatr Gastroenterol Nutr 1995;20:209-14.

7. Talley NJ, Phillips SF, Wiltgen CM, Zinsmeister AR, Melton LJ. Assessment of functional gastrointestinal disease: the bowel disease questionnaire. Mayo Clin Proc 1990;65:1456-79.

8. Walker LS, Caplan-Dover A, Rasquin-Weber A. Manual for the Questionnaire on Pediatric Gastrointestinal Symptoms [Thesis]. Nashville, TN: Vanderbilt University Department of Pediatrics; 2000.

9. National Center for Health Statistics (NCHS) 2000 [homepage na Internet]. CDC growth charts: United States. [atualizado em 27 de janeiro de 2007; citado em 6 de março de 2007]. Disponível em: http://www.cdc.gov/nchs/about/major/nhanes/growthcharts/datafiles.htm

10. Rasquin A, Di Lorenzo C, Forbes D, Guiraldes E, Hyams JS, Staiano A et al. Childhood functional gastrointestinal disorders: child/adolescent. Gastroenterology 2006;130:1527-37.

11. Baker SS, Liptak GS, Colletti RB, Croffie JM, Di Lorenzo C, Ector W et al. Constipation in infants and children: evaluation and treatment. A medical position statement of the North American Society for Pediatric Gastroenterology and Nutrition. J Pediatr Gastroenterol Nutr 1999;29:612-26.
12. Caplan A, Walker L, Rasquin A. Development and preliminary validation of the questionnaire on pediatric gastrointestinal symptoms to assess functional gastrointestinal disorders in children and adolescents. J Pediatr Gastroenterol Nutr 2005;41:296-304.

13. Croffie JM, Fitzgerald JF, Chong SK. Recurrent abdominal pain in children - a retrospective study of outcome in a group referred to a pediatric gastroenterology practice. Clin Pediatr (Phila) 2000;39:267-74.

14. Hyams JS, Davis P, Sylvester FA, Zeiter DK, Justinich CJ, Lerer T. Dyspepsia in children and adolescents: a prospective study. J Pediatr Gastroenterol Nutr 2000;30:413-8.

15. Miele E, Simeone D, Marino A, Greco L, Auricchio R, Novek SJ et al. Functional gastrointestinal disorders in children: an Italian prospective survey. Pediatrics 2004;114:73-8.

16. Edwards MC, Finney JW, Bonner M. Matching treatment with recurrent abdominal pain symptoms: an evaluation of dietary fiber and relaxation treatments. Behav Therapy 1991;22:257-67.

17. Chelimsky G, Czinn SJ. Techniques for the evaluation of dyspepsia in children J Clin Gastroenterol 2001;33:11-3.

18. Hyams JS, Davis P, Sylvester FA, Zeiter DK, Justinich CJ, Lerer T. Dyspepsia in children and adolescents: a prospective study. J Pediatr Gastroenterol Nutr 2000;30:413-8.

19. Gremse DA, Nguyenduc GH, Sacks AI, DiPalma JA. Irritable bowel syndrome and lactose maldigestion in recurrent abdominal pain in childhood. South Med J 1999;92:778-81.

20. Gremse DA, Sacks Al. Evaluation of dyspepsia. Pediatr Ann 1997;26:251-9.

21. El-Matary W, Spray C, Sandhu B. Irritable bowel syndrome: the commonest cause of recurrent abdominal pain in children. Eur J Pediatr 2004;163:584-8.

22. Toledo RJ. Má absorção de lactose em população de baixa renda. Suas implicações em um programa de suplementação alimentar com leite [tese de doutorado]. Campinas (SP): Faculdade de Ciências Médicas da Unicamp; 1984.

23. Koda YKL. Dor abdominal recorrente. In: Barbieri D, Koda YKL, editores. Doenças gastrenterológicas em pediatria. $1^{\mathrm{a}}$ ed. São Paulo: Atheneu; 1996. p. 412-8.

24. Burke P, Elliott M, Fleissner R. Irritable bowel syndrome and recurrent abdominal pain. A comparative review. Psychosomatics 1999;40:277-85. 